\title{
BMC Complementary and alternative medicine reviewer acknowledgements, 2014
}

Thomas A Rowles

\author{
Omar Abdel Salam \\ Egypt \\ Roslida Abdul Hamid \\ Malaysia
}

Aisha Abou Zeid

Egypt

Kamal-Eldin Abou-Elhamd

Egypt

Sawsan Abuhamdah
Jordan

Abuduaini Abulimiti

USA

Sandra Adams

USA

Salmon Adebayo

South Africa

Adewalw Adetutu

Nigeria

Oluyomi Adeyemi

Nigeria

Hadi Adibi

Iran

Neda Adibpour

Iran

Bulus Adzu

Nigeria

Abhinav Aeron
India

Eman Afify

Egypt

\author{
Muhammad Afzal \\ India
}

Gabriel Agbor

Cameroon

Bharat Aggarwal

USA

Bharat Aggarwal

USA

Riaz Ahmad

India

Sarfaraz Ahmad

USA

Amirhossein Ahmadi

Iran

Akefeh Ahmadiafshar

Iran

Augustine Ahmadu

Nigeria

Danish Ahmed

India

Khaled Ahmed

Malaysia

Mohamed Ahmed

Egypt

Osama Ahmed

Egypt

Kyung-Seop Ahn

South Korea

Mikel Aickin

USA
Nuriye Akev

Turkey

Dewan Akhter

Bangladesh

Hassan Akrami

Iran

Ali Al-Ahmad

Germany

Amin Alasbahi

Yemen

Angel Alex

India

Matthew Alexander

USA

Mohammed Al-Hariri

Saudi Arabia

Mahmoud Alhosin

France

Ehab Ali

Egypt

Nahid Ali

India

Niaz Ali

Pakistan

Md Ramjan Ali

Bangladesh

Mohammed Ali-Shtayeh

Palestinian Territory

Eman Alissa

Saudi Arabia

Correspondence: tom.rowles@biomedcentral.com

BioMed Central, Floor 6, 236 Gray's Inn Road, London WC1X 8HB, UK 


\begin{tabular}{|c|c|c|}
\hline $\begin{array}{l}\text { Aishah Al-Jarallah } \\
\text { Kuwait }\end{array}$ & $\begin{array}{l}\text { Jann Arends } \\
\text { Germany }\end{array}$ & $\begin{array}{l}\text { Mohammed Auwal Ibrahim } \\
\text { Nigeria }\end{array}$ \\
\hline $\begin{array}{l}\text { Angel Alonso } \\
\text { Mexico }\end{array}$ & $\begin{array}{l}\text { Ebru Arioglu Inan } \\
\text { Turkey }\end{array}$ & $\begin{array}{l}\text { Suresh Awale } \\
\text { Japan }\end{array}$ \\
\hline $\begin{array}{l}\text { Terje Alraek } \\
\text { Norway }\end{array}$ & $\begin{array}{l}\text { Nanfack Donfack Arno Rusel } \\
\text { Cameroon }\end{array}$ & $\begin{array}{l}\text { Maurice D Awouafack } \\
\text { Cameroon }\end{array}$ \\
\hline $\begin{array}{l}\text { Celuta Alviano } \\
\text { Brazil }\end{array}$ & $\begin{array}{l}\text { Ahmed Aroke Shahid } \\
\text { Nigeria }\end{array}$ & $\begin{array}{l}\text { Muhammad Ayaz } \\
\text { Pakistan }\end{array}$ \\
\hline $\begin{array}{l}\text { Adel Al-Zubairi } \\
\text { Saudi Arabia }\end{array}$ & $\begin{array}{l}\text { Ami Aronheim } \\
\text { Israel }\end{array}$ & $\begin{array}{l}\text { Boshra Azadi } \\
\text { Iran }\end{array}$ \\
\hline $\begin{array}{l}\text { Amro Amara } \\
\text { Egypt }\end{array}$ & $\begin{array}{l}\text { Protus Arrey Tarkang } \\
\text { Cameroon }\end{array}$ & $\begin{array}{l}\text { Ulken Tunga Babaoglu } \\
\text { Turkey }\end{array}$ \\
\hline $\begin{array}{l}\text { Angela Amedee } \\
\text { USA }\end{array}$ & $\begin{array}{l}\text { Myriam Arriaga-Alba } \\
\text { Mexico }\end{array}$ & $\begin{array}{l}\text { Sitesh C Bachar } \\
\text { Bangladesh }\end{array}$ \\
\hline $\begin{array}{l}\text { Mehrnoush Amid } \\
\text { Malaysia }\end{array}$ & $\begin{array}{l}\text { Majid Artus } \\
\text { United Kingdom }\end{array}$ & $\begin{array}{l}\text { Soo Kyung Bae } \\
\text { South Korea }\end{array}$ \\
\hline $\begin{array}{l}\text { Oluwatoyin Amira } \\
\text { Nigeria }\end{array}$ & $\begin{array}{l}\text { Kantha Deivi Arunachalam } \\
\text { India }\end{array}$ & $\begin{array}{l}\text { Yoe-Sik Bae } \\
\text { South Korea }\end{array}$ \\
\hline $\begin{array}{l}\text { Madhu Anand-Srivastava } \\
\text { Canada }\end{array}$ & $\begin{array}{l}\text { Mohammed Asad } \\
\text { Saudi Arabia }\end{array}$ & $\begin{array}{l}\text { Margarete Dulce Bagatini } \\
\text { Brazil }\end{array}$ \\
\hline $\begin{array}{l}\text { Shyam Sunder Anchuri } \\
\text { India }\end{array}$ & $\begin{array}{l}\text { Yoshihisa Asada } \\
\text { Japan }\end{array}$ & $\begin{array}{l}\text { Victor Bagla } \\
\text { South Africa }\end{array}$ \\
\hline $\begin{array}{l}\text { Joel Anderson } \\
\text { USA }\end{array}$ & $\begin{array}{l}\text { Atsushi Asakura } \\
\text { USA }\end{array}$ & $\begin{array}{l}\text { Puneet Kumar Bagri } \\
\text { India }\end{array}$ \\
\hline $\begin{array}{l}\text { Ana Andreazzi } \\
\text { Brazil }\end{array}$ & $\begin{array}{l}\text { George Asare } \\
\text { Ghana }\end{array}$ & $\begin{array}{l}\text { Jie Bai } \\
\text { China }\end{array}$ \\
\hline $\begin{array}{l}\text { Ofélia Anjos } \\
\text { Portugal }\end{array}$ & $\begin{array}{l}\text { Gary Asher } \\
\text { USA }\end{array}$ & $\begin{array}{l}\text { Yanhong Bai } \\
\text { China }\end{array}$ \\
\hline $\begin{array}{l}\text { Shahid Ansari } \\
\text { India }\end{array}$ & $\begin{array}{l}\text { Sadegh Ashkani } \\
\text { Malaysia }\end{array}$ & $\begin{array}{l}\text { Inkyung Baik } \\
\text { South Korea }\end{array}$ \\
\hline $\begin{array}{l}\text { Emmanouil Apostolidis } \\
\text { USA }\end{array}$ & $\begin{array}{l}\text { Mohamed Ashour } \\
\text { Egypt }\end{array}$ & $\begin{array}{l}\text { Vivek K. Bajpai } \\
\text { South Korea }\end{array}$ \\
\hline $\begin{array}{l}\text { Chippada Apparao } \\
\text { India }\end{array}$ & $\begin{array}{l}\text { Sinem Aslan Erdem } \\
\text { Turkey }\end{array}$ & $\begin{array}{l}\text { Usman Bala } \\
\text { Malaysia }\end{array}$ \\
\hline $\begin{array}{l}\text { Danielle Aragão } \\
\text { Brazil }\end{array}$ & $\begin{array}{l}\text { Özlem Sultan Aslantürk } \\
\text { Turkey }\end{array}$ & $\begin{array}{l}\text { Prabha Balaram } \\
\text { Malaysia }\end{array}$ \\
\hline $\begin{array}{l}\text { Sandra Arandelovic } \\
\text { Serbia }\end{array}$ & $\begin{array}{l}\text { Stelios Assimakopoulos } \\
\text { Greece }\end{array}$ & $\begin{array}{l}\text { Ewa Balcerczak } \\
\text { Poland }\end{array}$ \\
\hline $\begin{array}{l}\text { Jesil Aranjani } \\
\text { India }\end{array}$ & $\begin{array}{l}\text { Gilbert Ateufack } \\
\text { Cameroon }\end{array}$ & $\begin{array}{l}\text { Matthew Bambling } \\
\text { Australia }\end{array}$ \\
\hline $\begin{array}{l}\text { Adeyemi Oladapo Aremu } \\
\text { South Africa }\end{array}$ & $\begin{array}{l}\text { Ehud Atoun } \\
\text { Israel }\end{array}$ & $\begin{array}{l}\text { Heejung Bang } \\
\text { USA }\end{array}$ \\
\hline $\begin{array}{l}\text { Jann Arends } \\
\text { Germany }\end{array}$ & $\begin{array}{l}\text { Jithan Aukunuru } \\
\text { India }\end{array}$ & $\begin{array}{l}\text { Parveen Bansal } \\
\text { India }\end{array}$ \\
\hline
\end{tabular}


Yogendra Kumar Bansal

India

Jennifer Barber-Singh

USA

Sanaa Bardaweel

Jordan

Jo Barnes

New Zealand

Matthew Philip Greig Barnett

New Zealand

Luis Isamu Barros Kanzaki

Brazil

Silpi Basak

India

Ronan Batista

Brazil

Maurizio Battino

Italy

Saw Bawm

Myanmar

P S Bedi

India

Tania Begum

Sweden

Florian Beissner

Germany

Daniela Benedec

Romania

Kirsten Benkendorff

Australia

Alan Bensoussan

Australia

Iris Benzie

Hong Kong

Vance Berger

USA

Liron Berkovich

Israel

Dominique Bernard-Gallon

France

Luciano Bernardi

Italy

H Tanju Besler

Turkey
Thoudam Bhaigyabati

India

Supriya Bhalerao

India

Sowmya Bharani

United Kingdom

Khursheed Bhat

India

Piyali Bhattacharyya

Puerto Rico

Tapan Bhattacharyya

Afghanistan

Zhaoxiang Bian

Hong Kong

Stephen Birch

Netherlands

Tony Bird

Australia

Tannaz Birdi

India

Diane Birt

USA

Anupam Bishayee

USA

Felicity Bishop

United Kingdom

Anne Blais

France

Liesl Blott

Australia

Liu Bo

China

Ebru Bodur

Turkey

Friedrich Boege

Germany

Katja Boehm

Germany

Pompei Bolfa

Saint Kitts and Nevis

Maria Bonfleur

Brazil

Thidarut Boonmars

Thailand
Sandra Bosco

Brazil

R Beklem Bostancioglu

Turkey

Eliete Bouskela

Brazil

Fabrice Boyom

Cameroon

Fabrice Boyom

Cameroon

Kim Bridle

Australia

Guy Brock

USA

Julie Brown

New Zealand

Lindsay Brown

Australia

Iguatemy Lourenço Brunetti

Brazil

Angela Bruzzaniti

USA

Diego Bucci

Italy

Steven Budsberg

USA

Arndt Buessing

Germany

Latifa Bulbul

Bangladesh

Silvia Burlina

Italy

Andrea Cabarkapa

Serbia

Célia Cabral

Portugal

Jing Cai

China

Shi-Ying Cai

USA

Gul Sinem Cakir

Turkey

Gioacchino Calapai

Italy 
Jose Camara

Portugal

David Cameron-Smith

New Zealand

John Cannell

USA

Hongbo Cao

China

Huijuan Cao

China

Raffaele Capasso

Italy

Davie Cappoen

Belgium

Laura Carim Todd

USA

Klaudija Carovic-Stanko

Croatia

Judith Carroll

USA

Steven Cartwright

United Kingdom

Tina Cartwright

United Kingdom

John Cavanagh

USA

Carlos Ccavaleiro

Portugal

Ali Osman Ceribasi

Turkey

Rainer Cermak

Germany

Gayathri Chadalapaka

USA

Han Chae

South Korea

Younbyoung Chae

South Korea

Yubo Chai

USA

Kelvin Chan

Hong Kong

Yin-Ching Chan

Taiwan
Chanpen Chanchao

Thailand

Phool Chandra

India

Anish Chandy

Malaysia

Cheng-Shyong Chang

Taiwan

Fang-Rong Chang

Taiwan

Hsiao-Yun Chang

Taiwan

Hsueh-Wei Chang

Taiwan

Lee-Tian Chang

Taiwan

Mei Ying Chang

Taiwan

Shih-Liang Chang

Taiwan

Sungwon Chang

Australia

Weizhong Chang

USA

Ling Changquan

China

Pi Yu Chao

Taiwan

Srinivasa Chary

South Africa

Varanuj Chatsudthipong

Thailand

Farid Chemat

France

Wei Chen

China

Ching-Hsein Chen

Taiwan

Pei-Jie Chen

China

Yeng Chen

Malaysia

Chun-Jen Chen

Taiwan
Jing-Hsien Chen

Taiwan

Qingyong Chen

China

Fang-Pey Chen

Taiwan

Hubiao Chen

Hong Kong

Henian Chen

USA

Jiachun Chen

China

Jing Chen

USA

Jih-Jung Chen

Taiwan

Jaw-Wen Chen

Taiwan

Jianxin Chen

China

Pei-Ni Chen

Taiwan

Shih-Shun Chen

Taiwan

Lixin Chen

China

Xiuping Chen

Macao

Yangchao Chen

Hong Kong

Yu-Chun Chen

Taiwan

Chin-Yi Cheng

Taiwan

Juei-Tang Cheng

Taiwan

Jae Hoon Cheong

South Korea

Hsiu-Mei Chiang

Taiwan

Lucas Chibli

Brazil

Shih-Chang Chien

Taiwan 
Jin Han Chin

Malaysia

Siewmooi Ching

Malaysia

Salvatore Chirumbolo

Italy

Salvatore Chirumbolo

Italy

Chien Chih Chiu

Taiwan

Wanchun Chiu

Taiwan

Hye-Youn Cho

USA

William Cs Cho

Hong Kong

Won-Kyung Cho

South Korea

Yung Hyun Choi

South Korea

Sun-Mi Choi

South Korea

Shahabuddin Choudhuri

Bangladesh

Altino Choupina

Portugal

Nho Chu Won

South Korea

Lee Suan Chua

Malaysia

Anderly Chueh

Australia

Jiang Chunming

China

Martin Church

United Kingdom

Sasitorn Chusri

Thailand

Richard Cimanga Kanyanga

Belgium

Sofie Claerhout

Belgium

Sofie Clais

Belgium
Natalie Clark

USA

Yuri Clement

Trinidad and Tobago

Ignea Codruta

Greece

Steven Cok

USA

Talita Colomeu

Brazil

Violeta Colova-Tsolova

USA

Kieran Cooley

Canada

Edwin Cooper

USA

Werner Cordier

South Africa

Paola Costelli

Italy

Samantha Coulson

Australia

Virginia Cowen

USA

Holger Cramer

Germany

Helen Cramer

United Kingdom

Sybil Crawford

USA

Duncan Cromarty

South Africa

S Nicole Culos-Reed

Canada

Ray Cursons

New Zealand

Salvatore Cuzzocrea

Italy

Ademar Alves Da Silva Filho

Brazil

Sanja Dabelic

Croatia

Fulvio D'Acquisto

United Kingdom
Christopher D'Adamo

USA

Ning Dai

China

Andrew Dainty

United Kingdom

Abdulkarim Dakah

Syria

Archana Damle

India

Robert Dantzer

USA

Christophe Dardonville

Spain

Dana Darwish

Jordan

Undurti Das

USA

Alok Das Mohapatra

USA

Gouri Kumar Dash

Malaysia

Matheus Augusto De Bittencourt

Pasquali

Canada

Solange Lisboa De Castro

Brazil

Vincenzo De Feo

Italy

Jillian De Gezelle

USA

Harry De Koning

United Kingdom

Giuseppina De Petro

Italy

Damião De Sousa

Brazil

Lokesh Deb

India

Arnaud Dechamps

Indonesia

Felipe De-Faria

Brazil 
Sukanya Dej-Adisai

Thailand

Margarida Delgado

Portugal

Roxana Delgado

USA

Preeti Desai

India

Rahul Deshmukh

USA

Serawit Deyno

Ethiopia

Suranganie Dharmawardhane USA

Roberta Di Pietro

Italy

Teresa Dias

Portugal

Konstantinos Dimas

Greece

Laurence Dinan

United Kingdom

Pei-Hui Ding

China

Mohamed Dkhil

Saudi Arabia

Jingcheng Dong

China

Anton Dormer

USA

Patrick Dougherty

USA

Jurgen Drewe

Switzerland

Donna M Dryden

Canada

Dongshu Du

China

Jin Ao Duan

China

Nawal Dubey

India

Pierre Duez

Belgium
Heather Duncan

USA

Neil Duncan

South Africa

Veeramuthu Duraipandiyan

India

Elisângela Düsman

Brazil

Som Dutt

India

Abirlal Dutta

India

Chandradhar Dwivedi

USA

Gary Dykes

Malaysia

Jean Paul Dzoyem

Cameroon

Mohammad Ali Ebrahimzadeh

Iran

Padilla Eduardo

Mexico

Thomas Efferth

Germany

Brandon Eggleston

USA

Günter Eisele

Switzerland

Daisuke Ekuni

Japan

Rajaa El Bekay

Spain

Mohamed El Gendy

Canada

Sabry El-Bahr

Egypt

Fatma El-Demerdash

Egypt

Esam Elgorashi

South Africa

Ishaku Elisha

South Africa

Khaled El-Massry

Egypt
Nguelefack-Mbuyo Pami Elvine

Cameroon

Jurgen Engele

Germany

Ephrem Engidawork

Ethiopia

Shin Enosawa

Japan

Wan Kyu Eo

South Korea

Gokhan Eraslan

Turkey

Edzard Ernst

United Kingdom

Esra Eroglu Ozkan

Turkey

S Pelin Ertürküner

Turkey

Olorunfemi Eseyin

Nigeria

Manuel Estrada

Chile

Bruno Eto

France

Adaobi Ezike

Nigeria

Lucia Helena Faccioli

Brazil

Enrico Facco

Italy

Saudat Fadeyi

USA

Lihong Fan

China

Junming Fan

China

Yongjun Fan

Australia

Chee-Mun Fang

Malaysia

Jianqiao Fang

China

Jiliang Fang

China 


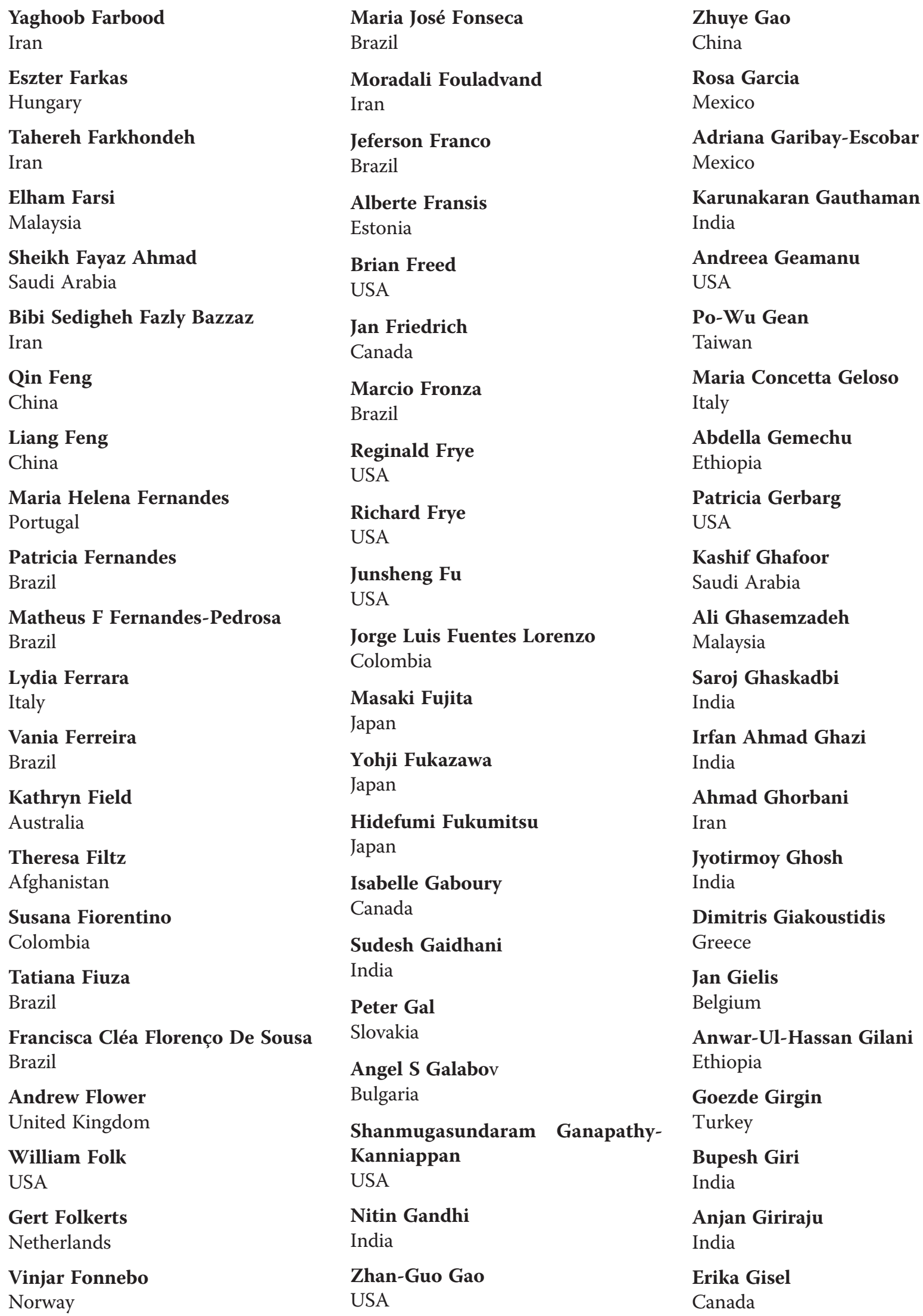




\begin{tabular}{|c|c|c|}
\hline $\begin{array}{l}\text { Andy Göbel } \\
\text { Germany }\end{array}$ & $\begin{array}{l}\text { Joao Bosco Guerreiro Da Silva } \\
\text { Brazil }\end{array}$ & $\begin{array}{l}\text { Richard Hammerschlag } \\
\text { USA }\end{array}$ \\
\hline $\begin{array}{l}\text { Amanuel Godebo } \\
\text { Ethiopia }\end{array}$ & $\begin{array}{l}\text { Corina Guethlin } \\
\text { Germany }\end{array}$ & $\begin{array}{l}\text { Jing-Yan Han } \\
\text { China }\end{array}$ \\
\hline $\begin{array}{l}\text { Bhomik Goel } \\
\text { India }\end{array}$ & $\begin{array}{l}\text { Gunjan Guha } \\
\text { USA }\end{array}$ & $\begin{array}{l}\text { Sangmi Han } \\
\text { South Korea }\end{array}$ \\
\hline $\begin{array}{l}\text { Antony Gomes } \\
\text { India }\end{array}$ & $\begin{array}{l}\text { Davide Guido } \\
\text { Italy }\end{array}$ & $\begin{array}{l}\text { Sang-Bae Han } \\
\text { South Korea }\end{array}$ \\
\hline $\begin{array}{l}\text { Nian Gong } \\
\text { USA }\end{array}$ & $\begin{array}{l}\text { Gaël Guilhem } \\
\text { France }\end{array}$ & $\begin{array}{l}\text { Sung Soo Han } \\
\text { South Korea }\end{array}$ \\
\hline $\begin{array}{l}\text { Zuojiong Gong } \\
\text { China }\end{array}$ & $\begin{array}{l}\text { Ilhami Gulcin } \\
\text { Turkey }\end{array}$ & $\begin{array}{l}\text { Manjunatha Hanumanthappa } \\
\text { India }\end{array}$ \\
\hline $\begin{array}{l}\text { Marie-Paule Gonthier } \\
\text { France }\end{array}$ & $\begin{array}{l}\text { Iram Gull } \\
\text { Pakistan }\end{array}$ & $\begin{array}{l}\text { Hideaki Hara } \\
\text { Japan }\end{array}$ \\
\hline $\begin{array}{l}\text { Margarete Goppelt-Struebe } \\
\text { Germany }\end{array}$ & $\begin{array}{l}\text { Songxue Guo } \\
\text { China }\end{array}$ & $\begin{array}{l}\text { Ulf Harnack } \\
\text { Germany }\end{array}$ \\
\hline $\begin{array}{l}\text { Johanna Gostner } \\
\text { Austria }\end{array}$ & $\begin{array}{l}\text { Xinfeng Guo } \\
\text { China }\end{array}$ & $\begin{array}{l}\text { Maryam Hassanzadeh Bashtian } \\
\text { Iran }\end{array}$ \\
\hline $\begin{array}{l}\text { Kazuo Goto } \\
\text { Japan }\end{array}$ & $\begin{array}{l}\text { Feifan Guo } \\
\text { China }\end{array}$ & $\begin{array}{l}\text { Cheryl Hawk } \\
\text { USA }\end{array}$ \\
\hline $\begin{array}{l}\text { Lingshan Gou } \\
\text { China }\end{array}$ & $\begin{array}{l}\text { Zhi-Ling Guo } \\
\text { USA }\end{array}$ & $\begin{array}{l}\text { Dongyuan He } \\
\text { China }\end{array}$ \\
\hline $\begin{array}{l}\text { Eman Gouda } \\
\text { Egypt }\end{array}$ & $\begin{array}{l}\text { Krishna Gupta } \\
\text { India }\end{array}$ & $\begin{array}{l}\text { Jun He } \\
\text { China }\end{array}$ \\
\hline $\begin{array}{l}\text { Mary Grace } \\
\text { USA }\end{array}$ & $\begin{array}{l}\text { Rajiv Gupta } \\
\text { India }\end{array}$ & $\begin{array}{l}\text { Lang He } \\
\text { China }\end{array}$ \\
\hline $\begin{array}{l}\text { Sandra Grace } \\
\text { Australia }\end{array}$ & $\begin{array}{l}\text { Saurabh Gupta } \\
\text { India }\end{array}$ & $\begin{array}{l}\text { Ulla Hedegaard } \\
\text { Denmark }\end{array}$ \\
\hline $\begin{array}{l}\text { Felix Grases } \\
\text { Spain }\end{array}$ & $\begin{array}{l}\text { Kitae Ha } \\
\text { South Korea }\end{array}$ & $\begin{array}{l}\text { Jodi Hedges } \\
\text { USA }\end{array}$ \\
\hline $\begin{array}{l}\text { Christopher Gray } \\
\text { Canada }\end{array}$ & $\begin{array}{l}\text { Ki-Chan Ha } \\
\text { South Korea }\end{array}$ & $\begin{array}{l}\text { Horacio Heinzen } \\
\text { Uruguay }\end{array}$ \\
\hline $\begin{array}{l}\text { Sara Greay } \\
\text { Australia }\end{array}$ & $\begin{array}{l}\text { Marwan Habiba } \\
\text { United Kingdom }\end{array}$ & $\begin{array}{l}\text { S Hemalatha } \\
\text { India }\end{array}$ \\
\hline $\begin{array}{l}\text { Joel Greenberger } \\
\text { USA }\end{array}$ & $\begin{array}{l}\text { Marshall Hagins } \\
\text { USA }\end{array}$ & $\begin{array}{l}\text { Ali Asghar Hemmati } \\
\text { Iran }\end{array}$ \\
\hline $\begin{array}{l}\text { Phillip Greenspan } \\
\text { USA }\end{array}$ & $\begin{array}{l}\text { Heidemarie Haller } \\
\text { Germany }\end{array}$ & $\begin{array}{l}\text { Rini Hendriani } \\
\text { Indonesia }\end{array}$ \\
\hline $\begin{array}{l}\text { Jeffrey Greeson } \\
\text { USA }\end{array}$ & $\begin{array}{l}\text { Khaled Hamden } \\
\text { Tunisia }\end{array}$ & $\begin{array}{l}\text { Ana Henriques } \\
\text { Portugal }\end{array}$ \\
\hline $\begin{array}{l}\text { Erik Groessl } \\
\text { USA }\end{array}$ & $\begin{array}{l}\text { Nadia Hamdy } \\
\text { Egypt }\end{array}$ & $\begin{array}{l}\text { Maria Das Graças Henriques } \\
\text { Brazil }\end{array}$ \\
\hline $\begin{array}{l}\text { Amelia Guadalupe-Grau } \\
\text { Denmark }\end{array}$ & $\begin{array}{l}\text { Ann Hamilton } \\
\text { USA }\end{array}$ & $\begin{array}{l}\text { Alberto Marcos Heredia Rizo } \\
\text { Spain }\end{array}$ \\
\hline
\end{tabular}


Mona Hetta

Egypt

Koji Higai

Japan

Clélia Akiko Hiruma-Lima

Brazil

Joanna Hlebowicz

Sweden

Johanna Hok

Sweden

Richard Holubkov

USA

Kam-Lun Ellis Hon

Hong Kong

Jin Tae Hong

South Korea

Mee Young Hong

USA

Mahmoud Hosseini

Iran

Hossein Hosseinzadeh

Iran

Wen-Chi Hou

Taiwan

Yih-Shou Hsieh

Taiwan

Hsin Hsiu

Taiwan

Shih-Lan Hsu

Taiwan

Jun-Te Hsu

Taiwan

Zhi-Qi Hu

China

Dongsheng $\mathbf{H u}$

China

Jiang $\mathbf{H u}$

China

Yingwei $\mathbf{H u}$

China

Yuanjie Hu

USA

Cheng-Yang Huang

Taiwan
Yi-Tsau Huang

Taiwan

Jinhai Huang

China

\section{Kun Huang}

USA

Mei-Hua Huang

USA

Shou-Hsien Huang

Taiwan

Wei Huang

USA

Xiaoxing Huang

China

Sona Hudecova

Slovakia

Jutta Huebner

Germany

John Hughes

United Kingdom

Mahmoud Huleihel

Israel

Lee Hullender Rubin

USA

Laith Hussain-Alkhateeb

Sweden

Minwoo Hwang

South Korea

Shinn-Jang Hwang

Taiwan

Jin Won Hyun

South Korea

Zein Ibrahim

Egypt

Savarimuthu Ignacimuthu India

Zullies Ikawati

Indonesia

Mohammad Zafar Imam

Bangladesh

Paulo Imamura

Brazil

Naoki Inagaki

Japan
Masatoshi Inden

Japan

Zafar Iqbal

Pakistan

Mehrdad Iranshahi

Iran

Yoshitaka Isaka

Japan

Liz Isenring

Australia

Amirul Islam

Bangladesh

Md Rafikul Islam

Bangladesh

Sabariah Ismail

Malaysia

Toshinori Ito

Japan

Kazuhisa Iwabuchi

Japan

Ezekiel Olugbenga Iwalewa

Nigeria

Vidhya Iyer

USA

Mohamed Jaabir

India

Eric Jacobson

USA

Yves Jacquot

France

Hartmut Jaeschke

USA

Sediqeh Jalali

Iran

Naveena Janakiram

USA

Hae-Dong Jang

South Korea

Su Hee Jang

South Korea

Insoo Jang

South Korea

Tae Jung Jang

South Korea 
Upali W Jayasinghe

Australia

Kamalan Jeevaratnam

Malaysia

Kristina Jenett-Siems

Germany

Myung-Shin Jeon

South Korea

Daewon Jeong

South Korea

Woo-Sik Jeong

South Korea

Minjeong Jeong

South Korea

Rajesh Kumar Jha

India

Guang Ji

China

Lin-Tao Jia

China

Yantao Jia

China

Han Jia

China

Hong Jiang

China

Weihua Jiang

USA

Meiyan Jiang

USA

Ligang Jie

China

Maria Adelina Jiménez-Arellanes

Mexico

Chengyun Jin

China

Tomoko Jippo

Japan

Stefanie Joos

Germany

Amita Joshi

India

Chintamani Joshi

India
Narendra Joshi

India

Swati Joshi-Barve

USA

Il Lae Jung

South Korea

Helgi Jung

Mexico

Suh-Hang Juo

Taiwan

Wan Jy

China

Shuk-Man Ka

Taiwan

Satyan Kalkunte

USA

Venkatesh Kamath Kamath

India

Yuto Kamei

Japan

Bernard Kamsu Foguem

France

Tae Jin Kang

South Korea

Ning Kang

China

Hee-Kyoung Kang

South Korea

Young-Hee Kang

South Korea

Yu-Hsun Kao

Taiwan

Panayotis Karayannakidis

Greece

David Katerere

South Africa

Ruchika Kaul-Ghanekar

India

Tejinder Kaur

India

Srini Kaveri

France

Eric Kelley

USA
William Kelly

New Zealand

Moira Kelly

United Kingdom

Deborah Kennedy

Canada

Janet Kern

USA

Paula Kersten

New Zealand

Rana Keyhanmanesh

Iran

Mohamed Khalil

Saudi Arabia

Murad Khan

Pakistan

Haroon Khan

Pakistan

Kainat Khan

India

Mohd Khan

South Africa

Muhammad Khan

Pakistan

Zakia Khanam

Malaysia

Mahnaz Khanavi

Iran

Alfi Khatib

Malaysia

Mahmoud Khattab

Egypt

Mohamed T Khayyal

Egypt

Elian Khazneh

Czech Republic

Nafiseh Khosravi

Iran

Ar Khuda-Bukhsh

India

Lik Voon Kiew

Malaysia

Sharon Kilbreath

Australia 
Dae-Ok Kim

South Korea

Hyung Sik Kim

South Korea

Hyeung-Rak Kim

South Korea

Hee Young Kim

South Korea

Hyunho Kim

South Korea

Hyun Jik Kim

South Korea

Won Ho Kim

South Korea

Ji Yeon Kim

South Korea

Joong-Sun Kim

South Korea

Jae Yong Kim

South Korea

Hojun Kim

South Korea

Jong Bin Kim

South Korea

Young-Min Kim

South Korea

Kun Hyung Kim

South Korea

Sun Kwang Kim

South Korea

Sung Kim

South Korea

Jong-Choon Kim

South Korea

Wook Kim

South Korea

Young-Cheul Kim

USA

Shintaro Kinugawa

Japan

Roman Kireev

Spain

Irina Kiseleva

Russian Federation
Navneet Kishore

South Africa

Anoop Kishore

India

Attila Kiss

Sweden

Soressa Kitessa

Australia

Nadja Klafke

Germany

Riana Kleynhans

South Africa

Helmut Klocker

Austria

Chun Hay Ko

Hong Kong

Kwangsuk Ko

South Korea

Biplob Koch

India

George Koffuor

Ghana

Itaru Kojima

Japan

Yoshihiro Komada

Japan

Michio Komai

Japan

Sureshbabu Kondaveeti

India

Ah-Ng Tony Kong

USA

Xiangying Kong

China

Sumet Kongkiatpaiboon

Thailand

Alice Kongsted

Denmark

Rituraj Konwar

India

Yun Hyung Koog

South Korea

Shigeru Kotake

Japan
Demetrios Kouretas

Greece

Stanisław Kowalski

Poland

Omer Koz

Turkey

Rajesh Kp

India

Sandra Kraljevic Pavelic

Croatia

Nataly Kravchenko-Balasha

Israel

Nandakumar Krishnadas

India

Agnete Egilsdatter Kristoffersen Norway

Anil Kumar

India

Akhilesh Kumar

India

Amit Kumar

India

Nitesh Kumar

India

Vikas Kumar

India

Rajesh Kumar

India

Dinesh Kumar

India

Ravi Kumara

India

Joydeb Kumar Kundu

Bangladesh

Vijayanarayana Kunhikatta India

Kazuhiro Kunimasa

Japan

Ramkumar Kunka Mohanram India

Ajaikumar Kunnumakkara

USA

Jinn Rung Kuo

Taiwan 
Sajeera Kupittayanant

Thailand

\section{Hokeun Kwon \\ USA}

Hokeun Kwon

USA

Jung-Taek Kwon

South Korea

Dong-Yeul Kwon

South Korea

Umar Kyari Sandabe

Nigeria

Giovanni Michele Lacalandra

Italy

Sara Ladu

Italy

Rene Lafont

France

Joao Lago

Brazil

Vicente Lahera

Spain

Lily Lai

United Kingdom

Jung Nien Lai

Taiwan

Kuang-Chi Lai

Taiwan

Namrita Lall

South Africa

Fanny Lalot

Switzerland

Ouattara Lamoussa Paul

Burkina Faso

Jean Langlois

France

George Lansner

USA

Li Xing Lao

Hong Kong

Malinee Laopaiboon

Thailand

Gee W Lau

USA
Nico Laubscher

South Africa

Romy Lauche

Germany

Horng-Liang Lay

Taiwan

Diamanto Lazari

Greece

Bombi Lee

South Korea

Meeyoung Lee

South Korea

Myeong Soo Lee

South Korea

Hyangsook Lee

South Korea

Jang-Hern Lee

South Korea

Tzung-Yan Lee

Taiwan

Kwang Ho Lee

South Korea

Soo Young Lee

South Korea

Huei-Jane Lee

Taiwan

Ju Ah Lee

South Korea

Min Won Lee

South Korea

Bong Hyo Lee

South Korea

Sanghoon Lee

South Korea

Chelsey Lemaster

USA

Wossenseged Lemma

Ethiopia

Ping-Chung Leung

Hong Kong

George Lewith

United Kingdom

Juan Li

China
Jie Li

China

Jing $\mathbf{L i}$

China

Hu Li

USA

Chuanfu Li

China

Chenrui Li

China

Kewei Li

China

Li Li

USA

Lingyun Li

China

Min Li

Hong Kong

Ping Li

China

Xuting Li

China

Ya-Jun Li

China

Yousheng Li

China

Yu Li

China

Zilong Li

China

Cheng-Rui Li

USA

Min Li

USA

Peng Li

USA

Shulin Li

USA

Tsai-Chung Li

Taiwan

Xin-Min Li

Canada

Yin Lian

China 
Fan-Rong Liang

China

Hui-Fen Liao

Taiwan

Xing Liao

United Kingdom

Byungmook Lim

South Korea

Yang Mooi Lim

Malaysia

Diingbo Lin

USA

Yin-Ku Lin

Taiwan

Annemette Bondo Lind

Denmark

Ulrike Lindequist

Germany

Ingrid Liodden

Norway

Ying-Ming Liou

Taiwan

Patricia Lisboa

Brazil

Alyson Littman

USA

Jun Liu

China

Chenghai Liu

China

Huiqing Liu

USA

Jianping Liu

China

Cun-Zhi Liu

China

Bo Liu

China

Hongbing Liu

China

Xusheng Liu

China

Quanhong Liu

China
Shuman Liu

China

Yi-Wen Liu

Taiwan

Richard Lobo

India

Dario Lofrumento

Italy

Carl Lombard

South Africa

Suguna Lonchin

India

Andrew Long

United Kingdom

Angela Lopes

Brazil

Guillermo Lopez

Mexico

Nattaya Lourith

Thailand

Brenda Lovell

Canada

Zhigang Lu

China

Qing-Yi Lu

USA

Weidong Lu

USA

Larissa Lucena Périco

Brazil

Iréne Lund

Sweden

Jian Luo

USA

Deyan Luo

China

Devaraj $M$

India

Choong Je Ma

South Korea

Chaomei Ma

China

Liang Ma

China
Bingji Ma

China

Ian Mackenzie

United Kingdom

Sarah Maddocks

United Kingdom

Lizandra Magalhães

Brazil

Pérola Magalhães

Brazil

Pamela Maher

USA

Narayan Maheswary

Bangladesh

Ayman Mahmoud

Egypt

Fawzi Mahomoodally

Mauritius

Cristiane Maia

Brazil

Arindam Maitra

India

Juraj Majtan

Slovakia

Tshepiso Jan Makhafola

South Africa

Suzana Makpol

Malaysia

N Malangu

South Africa

Tauseef Malik

India

Mao-Qiang Man

USA

Thamilvaani Manaharan

Malaysia

Azadeh Manayi

Iran

Mahitosh Mandal

India

Soma Mandal

Canada

Santi M Mandal

India 
Melânia Manfron

Brazil

Supachoke Mangmool

Thailand

Tran Manh Hung

Viet Nam

Luigi Manni

Italy

Sharif Mansor

Malaysia

Jose Mansure

Canada

Isabelle Marc

Canada

Cristina Marcucci

Brazil

Donald Marcus

USA

Abdalbasit Mariod

Sudan

Jacquelyn Marsh

Canada

Wolfgang Marx

Australia

Robert Mathie

United Kingdom

Vitor Mati

Brazil

Ivana Matic

Serbia

Sannah Mativandlela

South Africa

Hisashi Matsuda

Japan

Sithandiwe Mazibuko

South Africa

Kishor Mazumder

Bangladesh

Karalyn Mcdonald

Australia

Lyndy Mcgaw

South Africa

Jane Mchowat

USA
Ahmed Mediani

Algeria

Eugene Megnassan

Cote d'Ivoire

Hayelom Mekonen

Ethiopia

Lighua Meng

China

Zhi-Hong Meng

China

Francesca Menniti

Italy

Filipe Mergulhao

Portugal

Francois Meurens

Canada

Marion Meyer

South Africa

Rikke Louise Meyer

Denmark

Sascha Meyer

Germany

Oliver Micke

Germany

Maria Miguel

Portugal

Marius Mihasan

Romania

Jessica Miller

USA

Mladen Milos

Croatia

Selvaraj Miltonprabu

India

Hyeyoung Min

South Korea

Do Sik Min

South Korea

Junki Min

South Korea

Bi Minggang

China

Silvano Mior

Canada
Maria Leticia Miranda Fernandes

Estevinho

Portugal

Shingen Misaka

Japan

Ashwini Mishra

USA

Megha Mittal

India

Tempei Miyaji

Japan

Shingo Miyata

Japan

Shingo Miyata

Japan

Wataru Mizunoya

Japan

Unnikrishnan Mk

India

Andrei Mocan

Romania

Reda Mohammed

Egypt

Maradumane Mohan

USA

Ayaz Mohd

Oman

Nurul 'Izzah Mohd Sarmin

Malaysia

Yasmin Anum Mohd Yusof

Malaysia

Alex Molassiotis

Hong Kong

Fernando P Molina-Heredia

Spain

Marisa Moller Wolmarans

South Africa

Robson Monteiro

Brazil

Lianet Monzote

Cuba

Michael Moore

United Kingdom 
Nicholas Moore

France

Alex Moroz

USA

Jane Morrell

Sweden

Mohamed Morsy

Egypt

Said Moselhy

Saudi Arabia

Abdel-Tawab Mossa

Egypt

Ramzi Mothana

Saudi Arabia

Andrea Motoyama

Brazil

Andrea Motta

Italy

Raymond Simplice Mouokeu

Cameroon

Mack Moyo

South Africa

Jayesh Mudgal

India

Karsten Muenstedt

Germany

Aliyu Muhammad

Nigeria

Naveed Muhammad

Pakistan

Faqir Muhammad

USA

Kalpana Mujoo

USA

Lillian Mukandiwa

South Africa

Rofhiwa Bridget Mulaudzi

South Africa

Eshetu Mulisa

Ethiopia

Niki Munk

USA

Renuka Munshi

India
Akira Murakami

Japan

Tsugiya Murayama

Japan

Frauke Musial

Norway

Michihiro Mutoh

Japan

Peter Mwitari

Kenya

Chang-Seon Myung

South Korea

Mahadevan N

India

Kesara Na-Bangchang

Thailand

Seyed Mohammad Nabavi

Iran

Pratibha Nadig

India

Richard J Naftalin

United Kingdom

Raghuram Nagarathna

India

Basavraj Nagoba

India

Vinny Naidoo

South Africa

Umberin Najeeb

Canada

Toshiaki Nakano

Taiwan

Ayse Nalbantsoy

Turkey

Donghyun Nam

South Korea

Kim Nam-Hee

South Korea

Madhavan Nampoothiri

India

Betty Namuddu

Uganda

Mohsen Naseri

Iran
Andrés Navarrete

Mexico

Yogendra Nayak

India

Yogendra Nayak

India

Ashwell Ndhlala

South Africa

Dieudonne Ndjonka

Cameroon

Candace Necyk

Canada

Bimla Nehru

India

Fatemeh Nejatzadeh

Iran

Telesphore Benoit Nguelefack

Cameroon

Endalkachew Nibret

Ethiopia

Glenda Nicioli Da Silva

Brazil

Loredan Stefan Niculescu

Romania

Akio Niimi

Japan

Nik Mohd Afizan Nik Abd. Rahman

Malaysia

Cristina Nogueira

Brazil

Fabiana Nonato

Brazil

Cesar Nopo-Olazabal

USA

Hedvig Nordeng

Norway

Farah Noureen

Pakistan

Maja Nowakowski

USA

Fidele Ntie-Kang

Cameroon

Lorena Nunez

South Africa 
Mohd Fahami Nur Azlina

Malaysia

Armania Nurdin

Olawole Obembe

Nigeria

Erica Oberg

USA

Kylie O'Brien

Australia

Young Ock Kim

South Korea

José-Enrique O'Connor

Spain

Olabisi Oduwole

Nigeria

Junetsu Ogasawara

Japan

Haruko Ogawa

Japan

Adenike Ogunshe

Nigeria

Oluwafemi Oguntibeju

South Africa

Byeongsang Oh

Australia

Myung Sook Oh

South Korea

Shreesh Ojha

United Arab Emirates

Yoshihiro Okamoto

Japan

Charles Okoli

Nigeria

Marina Okoshi

Brazil

Theophine Okoye

Nigeria

Tadayoshi Okumura

Japan

Toshikatsu Okumura

Japan

Olumayokun Olajide

United Kingdom
Oyinlola Olaokun

South Africa

Bogdan Olenyuk

USA

Maira Souza Oliveira

Brazil

Horacio Olivo

USA

Edwin Omeje

Nigeria

Tonia Onyeka

Nigeria

Nor Hayati Othman

Malaysia

Bamidele Owoyele

Nigeria

Ademola Oyagbemi

Nigeria

Marcin Ozarowski

Poland

Yoko Ozawa

Japan

Cristina Pacheco Soares

Brazil

Rabindra Padhy

India

Alessio Paffoni

Italy

Aravinda Pai

India

Poungrat Pakdeechote

Thailand

Shanthi Palanivelu

India

Sarawoot Palipoch

Thailand

Fabricio Pamplona

Brazil

Tai-Long Pan

Taiwan

Abhay K. Pandey

India

Siyaram Pandey

Canada
Perumal Pandurangan

India

Jong-Hwei Pang

Taiwan

Xiufeng Pang

China

Manju Panghal

India

Li-Heng Pao

Taiwan

Wansu Park

South Korea

Heonyong Park

South Korea

Jong Park

USA

Jongbae Park

USA

Kye Won Park

South Korea

Chan-Hong Park

South Korea

Seong-Uk Park

South Korea

Yongsoon Park

South Korea

Donna Parker

USA

Kyung-Hyun Park-Min

USA

Visweswara Rao Pasupuleti

Malaysia

Vinood Patek

United Kingdom

Paras Patel

India

Ravi Patel

USA

Charlotte Paterson

United Kingdom

Ashish Pathak

USA

Srinivas Patnala

South Africa 


\begin{tabular}{|c|c|c|}
\hline $\begin{array}{l}\text { Alexandra Paulo } \\
\text { Portugal }\end{array}$ & $\begin{array}{l}\text { Alberto Porta } \\
\text { Italy }\end{array}$ & $\begin{array}{l}\text { Batool Rahmati } \\
\text { Iran }\end{array}$ \\
\hline $\begin{array}{l}\text { Maxime Pellegrin } \\
\text { Switzerland }\end{array}$ & $\begin{array}{l}\text { Fernanda Portaro } \\
\text { Brazil }\end{array}$ & $\begin{array}{l}\text { Mohammad Rahmati Yamchi } \\
\text { Iran }\end{array}$ \\
\hline $\begin{array}{l}\text { Wenbo Peng } \\
\text { Australia }\end{array}$ & $\begin{array}{l}\text { Jalal Pourahmad } \\
\text { Iran }\end{array}$ & $\begin{array}{l}\text { Mohammed Rahmatullah } \\
\text { Bangladesh }\end{array}$ \\
\hline $\begin{array}{l}\text { Philip Peplow } \\
\text { New Zealand }\end{array}$ & $\begin{array}{l}\text { Spyros Pournaras } \\
\text { Greece }\end{array}$ & $\begin{array}{l}\text { Revathi Rajappan } \\
\text { India }\end{array}$ \\
\hline $\begin{array}{l}\text { Andrés Pereañez } \\
\text { Colombia }\end{array}$ & $\begin{array}{l}\text { Kirti Prabhu } \\
\text { India }\end{array}$ & $\begin{array}{l}\text { Nutan Rajput } \\
\text { India }\end{array}$ \\
\hline $\begin{array}{l}\text { Rosa M Perez-Gutierrez } \\
\text { Mexico }\end{array}$ & $\begin{array}{l}\text { John Preshanth Kumar } \\
\text { India }\end{array}$ & $\begin{array}{l}\text { Harinantenaina Rakotondraibe } \\
\text { USA }\end{array}$ \\
\hline $\begin{array}{l}\text { Cynthia Peterson } \\
\text { Switzerland }\end{array}$ & $\begin{array}{l}\text { Matthias Preusser } \\
\text { Austria }\end{array}$ & $\begin{array}{l}\text { Aswatha Ram H.N. } \\
\text { India }\end{array}$ \\
\hline $\begin{array}{l}\text { Kenneth Pfarr } \\
\text { Germany }\end{array}$ & $\begin{array}{l}\text { Sarah Price } \\
\text { United Kingdom }\end{array}$ & $\begin{array}{l}\text { Venkatesha Perumal Ramachandran } \\
\text { India }\end{array}$ \\
\hline $\begin{array}{l}\text { Ngoc Minh Pham } \\
\text { Viet Nam }\end{array}$ & $\begin{array}{l}\text { Gerhard Prinsloo } \\
\text { South Africa }\end{array}$ & $\begin{array}{l}\text { Vadde Ramakrishna } \\
\text { India }\end{array}$ \\
\hline $\begin{array}{l}\text { Mallikarjuna Rao Pichika } \\
\text { Malaysia }\end{array}$ & $\begin{array}{l}\text { Long Qu } \\
\text { USA }\end{array}$ & $\begin{array}{l}\text { Ravindhran Ramalingam } \\
\text { India }\end{array}$ \\
\hline $\begin{array}{l}\text { Anatole Constant Pieme } \\
\text { Cameroon }\end{array}$ & $\begin{array}{l}\text { Sara Quandt } \\
\text { USA }\end{array}$ & $\begin{array}{l}\text { Surash Ramanathan } \\
\text { Malaysia }\end{array}$ \\
\hline $\begin{array}{l}\text { Karen Pilkington } \\
\text { United Kingdom }\end{array}$ & $\begin{array}{l}\text { Cassandra Quave } \\
\text { USA }\end{array}$ & $\begin{array}{l}\text { Sujatha Ramasamy } \\
\text { Malaysia }\end{array}$ \\
\hline $\begin{array}{l}\text { Shubhangi Pingle } \\
\text { India }\end{array}$ & $\begin{array}{l}\text { Annie Quignard-Boulange } \\
\text { France }\end{array}$ & $\begin{array}{l}\text { Radhakrishnan Rameshkumar } \\
\text { India }\end{array}$ \\
\hline $\begin{array}{l}\text { Somchai Pinlaor } \\
\text { Thailand }\end{array}$ & $\begin{array}{l}\text { Jael Quintero } \\
\text { Mexico }\end{array}$ & $\begin{array}{l}\text { Sonia Ramos } \\
\text { Spain }\end{array}$ \\
\hline $\begin{array}{l}\text { Fabio Pittella Silva } \\
\text { Brazil }\end{array}$ & $\begin{array}{l}\text { Raymond Quock } \\
\text { USA }\end{array}$ & $\begin{array}{l}\text { Anand Ramteke } \\
\text { India }\end{array}$ \\
\hline $\begin{array}{l}\text { Marcia Regina Piuvezam } \\
\text { Brazil }\end{array}$ & $\begin{array}{l}\text { Rahmatullah Qureshi } \\
\text { Pakistan }\end{array}$ & $\begin{array}{l}\text { Ruiqiong Ran } \\
\text { USA }\end{array}$ \\
\hline $\begin{array}{l}\text { Maribel Plascencia-Jatomea } \\
\text { Mexico }\end{array}$ & $\begin{array}{l}\text { Karim Raafat } \\
\text { Lebanon }\end{array}$ & $\begin{array}{l}\text { Chandana Rao } \\
\text { India }\end{array}$ \\
\hline $\begin{array}{l}\text { Adrian Martin Pohlit } \\
\text { Brazil }\end{array}$ & $\begin{array}{l}\text { Mohamed Rabeh } \\
\text { Egypt }\end{array}$ & $\begin{array}{l}\text { Iraj Rasooli } \\
\text { Iran }\end{array}$ \\
\hline $\begin{array}{l}\text { Bernard Poitevin } \\
\text { France }\end{array}$ & $\begin{array}{l}\text { Gandhi Radis-Baptista } \\
\text { Brazil }\end{array}$ & $\begin{array}{l}\text { Zahid Rasul } \\
\text { France }\end{array}$ \\
\hline $\begin{array}{l}\text { Ganesan Ponesakki } \\
\text { India }\end{array}$ & $\begin{array}{l}\text { Atiar Rahman } \\
\text { Bangladesh }\end{array}$ & $\begin{array}{l}\text { Sudipt Rath } \\
\text { India }\end{array}$ \\
\hline $\begin{array}{l}\text { Beaudelaire Ponou Kemvoufo } \\
\text { Cameroon }\end{array}$ & $\begin{array}{l}\text { Md Masudur Rahman } \\
\text { Bangladesh }\end{array}$ & $\begin{array}{l}\text { Edward Ratovitski } \\
\text { USA }\end{array}$ \\
\hline $\begin{array}{l}\text { Antony Porcino } \\
\text { Canada }\end{array}$ & $\begin{array}{l}\text { Najm-Ur Rahman } \\
\text { Pakistan }\end{array}$ & $\begin{array}{l}\text { Jo-Anne Rayner } \\
\text { Australia }\end{array}$ \\
\hline
\end{tabular}


Daniel Redwood

USA

Ralf Regenthal

Germany

Juliana Quero Reimão

Brazil

Thomas Reinhold

Germany

Flavia Aparecida Resende

Brazil

Morayma Reyes

USA

Jennifer Rioux

USA

Cheryl Ritenbaugh

USA

Syed Ibrahim Rizvi

India

Pierre-Yves Rodondi

Switzerland

Juan Francisco Rodríguez-Landa

Mexico

Victor Romanov

USA

Irma Romero

Mexico

Philippe Rondeau

France

Ruiming Rong

China

Pornpimol Rongnoparut

Thailand

Elaine Rosas

Brazil

Ema Rosas-Burgos

Mexico

Bjorn Rosengren

Sweden

Rina Rosin-Arbesfeld

Israel

Anastasia Rowland-Seymour

USA

Aditi Roy

India
Abdur Rub

India

Eduardo Ruiz-Bustos

Mexico

Bernhard Ryffel

France

Jong Hoon Ryu

South Korea

Bashar Saad

Israel

Niina Saarinen

Finland

Maria Jose Saavedra

Portugal

Masoumeh Sabetkasaei

Iran

Katia Sabino

Brazil

Sherry Sachdeva

India

Azmi Johari Saiful

Malaysia

M Ram Sairam

Canada

Cagri Sakalar

Turkey

Meena Sakharkar

Japan

Hossam Salama

Egypt

Rciardo Salazar

Mexico

Sabita Saldanha

USA

Ammar Saleem

Canada

Avni Sali

Australia

Avni Sali

Australia

Naguib Salleh

Malaysia

Jagdeep Sandhu

Canada
Louis Pergaud Sandjo

Cameroon

Senthil Kumar Sankareswaran

India

Enrica Santarcangelo

Italy

Juan F Santibanez

Serbia

Rogerio Saraiva

Brazil

Sudarsini Saravanabhavan

Saudi Arabia

Moklesur Sarker

Japan

Bruno Sarmento

Portugal

Jerome Sarris

Australia

Masa Sasagawa

USA

Jiichiro Sasaki

Japan

Sreenivasan Sasidharan

Malaysia

Hiromi Sato

Japan

Neeraj Saxena

USA

Donna Scarborough

USA

Elad Schiff

Israel

Sallie Schneider

USA

Dominik Schöndorf

Germany

Robert Schulman

USA

Elita Scio

Brazil

Marília Seelaender

Brazil

Shuichi Segawa

Japan 
Veronique Seidel

United Kingdom

Shamala Devi Sekaran

Malaysia

Sonal Sekhar

India

Samy Selim

Egypt

Jasmine Selvakumar

India

Jonathan Sembrano

USA

David Senchina

USA

Chaminda Jayampath Seneviratne

Singapore

Surojeet Sengupta

USA

Dong-Wan Seo

South Korea

Byung-Kwan Seo

South Korea

Sonia Sequeira

USA

Jadranka Sertic

Croatia

Ben Sessa

United Kingdom

Robert Sewell

United Kingdom

Nadia Shaban

Egypt

Nermeen Shaffie

Egypt

Abdus Saboor Shah

China

Zahoor Shah

USA

Mohammad Reza Shakibaie

Iran

Baoci Shan

China

Hongcai Shang

China
Muthu Shanmugam

India

Jiangang Shen

Hong Kong

Xueyong Shen

China

Gajanan Sherbet

United Kingdom

Dazhuo Shi

China

Xianbao Shi

China

Workineh Shibeshi

Ethiopia

Su Shi-Bing

China

Jamil Shilpi

Bangladesh

Insop Shim

South Korea

Kazunori Shimada

Japan

Byung-Cheul Shin

South Korea

In Sik Shin

South Korea

Thimmappa Shivanandappa

India

Yun Hee Shon

South Korea

Mohamed Shoukri

Saudi Arabia

Yukihiro Shoyama

Japan

Richa Shri

India

Shruti Shukla

South Korea

Ines Sifaoui

Tunisia

Parames Sil

India

Teresinha Silva

Brazil
Edgar Silveira

Brazil

Ana Paula Simões-Wüst

Switzerland

Amanda Simons

USA

Maree Simpson

Australia

Zia-Ud-Din Sindhu

Pakistan

Harminder Singh

India

Nihar Singh

India

Pankaj Singh

USA

Rekha Singh

USA

Sarman Singh

India

Mukeshj Singh

India

Neeraj Sinha

India

Sivaperumal Sivaramakrishnan

India

Dan Sliva

USA

Caroline A Smith

Australia

Karen Snider

USA

Luiz Soares

Brazil

Stephanie Sohl

USA

Didem Sohretoglu

Turkey

Eeva Sointu

USA

Rosa Solã

Spain

Elizabeth Soliday

USA 


\begin{tabular}{|c|c|c|}
\hline Maha Soltan & Marius Stefan & Yoo-Hun Suh \\
\hline Egypt & Romania & South Korea \\
\hline Rakesh Somani & Christopher Stevenson & Kyoungho Suk \\
\hline India & Australia & South Korea \\
\hline Sivagurunathan Somasundaram & Joanna Stewart & Shaida Fariza Sulaiman \\
\hline USA & New Zealand & Malaysia \\
\hline Avinash Sonawane & Emilie Stolarczyk & Shubhankar Suman \\
\hline India & United Kingdom & USA \\
\hline Tuzz-Ying Song & David Stresser & Peng Sun \\
\hline Taiwan & USA & USA \\
\hline Hong-Li Song & Trine Stub & Xuejun Sun \\
\hline China & Norway & China \\
\hline Jianguo Song & Chun-Li Su & Tobias Sundberg \\
\hline China & Taiwan & Sweden \\
\hline Yiqing Song & Jason Su & Sugandhika Suresh \\
\hline USA & USA & Sri Lanka \\
\hline Germain Sotoing Taïwe & Ming-Jai Su & TA Ajith \\
\hline Cameroon & Taiwan & India \\
\hline Ilavenil Soundharrajan & Chin-Cheng Su & Hend Tag \\
\hline South Korea & Taiwan & Egypt \\
\hline Lauro Souza & Chun-Xiang Su & Reina Taguchi \\
\hline Brazil & China & Japan \\
\hline Preethi Soysa & $\mathrm{Zi}-\operatorname{Ren} \mathrm{Su}$ & Reinaldo Takahashi \\
\hline Sri Lanka & China & Brazil \\
\hline Claire Spears & Alírica Suárez & Hiroshi Takeda \\
\hline USA & Venezuela & Japan \\
\hline Mariana Spetea & Parasuraman Subramani & Kazuhiro Takemoto \\
\hline Austria & Malaysia & Japan \\
\hline Sarangapani Sreelatha & Mahesh Subramanian & Laura Talarico \\
\hline Singapore & India & Argentina \\
\hline Bungorn Sripanidkulchai & Sorimuthu Subramanian & Steve Talcott \\
\hline Thailand & India & USA \\
\hline Rungrudee Srisawat & Appian Subramoniam & Jean De Dieu Tamokou \\
\hline Thailand & India & Cameroon \\
\hline Anshu Srivastava & Ganapasam Sudhandiran & Hiroyuki Tanaka \\
\hline India & India & Japan \\
\hline Milan Stankovic & Jau-Ling Suen & Chanderdeep Tandon \\
\hline Serbia & Taiwan & India \\
\hline Amie Steel & Ivana Suffredini & Tao Tang \\
\hline Australia & Brazil & China \\
\hline Elizabeth Steels & Hong-Won Suh & Suni Tang \\
\hline Australia & South Korea & USA \\
\hline Vanessa Steenkamp & Hyung Joo Suh & Wei Tang \\
\hline South Africa & South Korea & Japan \\
\hline
\end{tabular}


Yong Tang

China

Yaxiong Tang

China

Yi Tang

China

Lijian Tao

China

Arda Tasatargil

Turkey

Anil Tatiya

India

Aldo Tava

Italy

Zahra Tayarani-Najaran

Iran

Ann Taylor

USA

Lisa Taylor-Swanson

USA

Gerald Ngo Teke

Cameroon

Gerald Ngo Teke

Cameroon

Nitin Telang

USA

Shirley Telles

India

Rémy Bertrand Teponno

Cameroon

Masanori Terasaki

Japan

Michael Teut

Germany

Supinya Tewtrakul

Thailand

Nai-Peng Tey

Malaysia

Nooruddin Thajuddin

India

Lal Thapa

Nepal

Kalaivani Thiagarajan

India
George Thomson

United Kingdom

Derun Tian

China

Stephanie Tjen-A-Looi

USA

Anil Kumar Tomar

India

Omer Toprak

Turkey

Masaru Toriyama

Japan

Marcio Torsoni

Brazil

Santoshkumar Tota

India

Oliver Treeck

Germany

Debu Tripathy

USA

Boryana Trusheva

Bulgaria

Fu-Ming Tsai

Taiwan

Chin-Chuan Tsai

Taiwan

Katrina Tsang

Hong Kong

William Tsang

China

Karl Wk Tsim

China

Pengfei Tu

China

Xiang Tu

China

Rajkumar Tulsawani

India

Shaikh Jamal Uddin

Bangladesh

Carlos Ueira-Vieira

Brazil

Rizwan Ul Haq

Pakistan
Dana Ullman

USA

Virgilijus Uloza

Lithuania

Jae-Young Um

South Korea

Padmaja Vaidyanathan

India

Abedin Vakili

Iran

Ben Valdez

USA

Dora Valencia

Mexico

Marieke Van Puymbroeck

USA

Jacob Van Tonder

South Africa

Sandy Van Vuuren

South Africa

Candice Van Wyk

South Africa

Robyn Van Zyl

South Africa

Ina Vandebroek

USA

Luca Vannucci

Czech Republic

Seda Vatansever

Turkey

Dhiraj Vattem

USA

Mahdi Vazirian

Iran

Krishna Kumar Veeravalli

USA

Ramaprabhu Vempati

India

Chandana Venkateswara Rao

India

Monika Vishnoi

USA

G L Viswanatha

India 
Luis Vitetta

Australia

Jiri Vrba

Czech Republic

Vladimir Vuksan

Canada

James Wachira

USA

Shelli Waetzig

USA

Shinya Wakusawa

Japan

Ju-Bo Wang

China

Bin Wang

USA

Ronald Wang

Hong Kong

Xiaoge Wang

China

Chong-Zhi Wang

USA

Fengshan Wang

China

Junpeng Wang

USA

Jian Wang

China

Shu-Ming Wang

USA

Shusheng Wang

USA

Guangjun Wang

China

Xiao Wang

China

Xiao-Ning Wang

China

Yinye Wang

China

Yingwei Wang

China

Wei Wang

USA
Zhijun Wang

USA

Jon Wardle

Australia

Narelle Warren

Australia

Ramida Watanapokasin

Thailand

Pierre Watcho

Cameroon

Chatchai Wattanapiromsakul

Thailand

John Weber

Canada

Laura Weeks

Canada

Jiang Wei

China

Ying Wei

China

Xiaolin Wei

China

Kuo-Ching Wen

Taiwan

Zhining Wen

China

Fu-Qiang Wen

China

Chia-Jui Weng

Taiwan

Ursula Werneke

Sweden

Maria Fernanda Werner

Brazil

Janis Whitlock

USA

Barbara Wider

United Kingdom

C Dilrukshi Wijayarathna

Sri Lanka

Jenny Wilkinson

Australia

Sonia Will

Brazil
Patrick Wilson

USA

Ursula Wolf

Switzerland

Benjamas Wongsatayanon

Thailand

Lisa Wood

USA

Julia Woodman

United Kingdom

Liming Wu

China

Chung-Hsuen Wu

Taiwan

Ming-Jiuan Wu

Taiwan

Ching-Shuang Wu

Taiwan

Ming Wu

USA

Tie Wu

China

Leder Xavier

Brazil

Chunmei Xia

China

Daozong Xia

China

Jing Xianghong

China

Xiao He Xiao

China

Cheng Xiao

China

Xiaohe Xiao

China

Yin Xiao

Australia

Linshen Xie

China

Wei-Fen Xie

China

Ye Xing-Ming

China 


\begin{tabular}{|c|c|c|}
\hline Xingjiang Xiong & Zhang Yanjun & Yuan Yuan \\
\hline China & China & China \\
\hline Guangwu Xiong & Wei Boon Yap & Jong Won Yun \\
\hline China & Malaysia & South Korea \\
\hline Xin-Gui Xiong & Jwu-Lai Yeh & Andrey Yurkov \\
\hline China & Taiwan & Germany \\
\hline Zhang Xm & Feng-Lin Yen & Yasim Yusof \\
\hline China & Taiwan & Malaysia \\
\hline Xudong Xu & Hung-Rong Yen & Zhonglin Yang \\
\hline China & Taiwan & China \\
\hline Lin $\mathrm{Xu}$ & Louis Yen & Fatheya Zahran \\
\hline Hong Kong & USA & Saudi Arabia \\
\hline Zhaohui Xu & Rakiswendé Serge Yerbanga & Bojan Zalar \\
\hline China & Burkina Faso & Slovenia \\
\hline Qianqian Xu & Beow Chin Yiap & Alireza Zamani \\
\hline China & Malaysia & Iran \\
\hline Shi-Wen Xu & Liusong Yin & Aleksander Zampronio \\
\hline China & USA & Brazil \\
\hline Bin $\mathrm{Xu}$ & Yanyan Yin & Nelo Zanchi \\
\hline China & China & Brazil \\
\hline Nik Soriani Yaacob & Masahiro Yoneda & Antonia Zapantis \\
\hline Malaysia & Japan & USA \\
\hline Toshihiko Yada & Audrey Yong & Zied Zarai \\
\hline Japan & Malaysia & Tunisia \\
\hline Arun Yadav & Seong Woo Yoon & Xiao Zhai \\
\hline India & South Korea & China \\
\hline Toru Yago & Takemi Yoshida & Yan Zhang \\
\hline Japan & Japan & China \\
\hline Badrul Yahaya & Zongbing You & Yongyu Zhang \\
\hline Malaysia & USA & China \\
\hline Sien-Hung Yang & Junsang Yu & Min Zhang \\
\hline Taiwan & South Korea & USA \\
\hline Guo-Yuan Yang & Wen-Jen Yu & Ruixin Zhang \\
\hline China & Taiwan & USA \\
\hline Wen-Chin Yang & Shu Juan Yu & Wei-Dong Zhang \\
\hline Taiwan & China & China \\
\hline Woong Mo Yang & Jianbo Yu & Xuemei Zhang \\
\hline South Korea & China & China \\
\hline Cai-Guang Yang & Ying Yu & Yan Zhang \\
\hline China & China & USA \\
\hline Guangxiao Yang & Zhi-Ling Yu & Ling Zhang \\
\hline China & Hong Kong & USA \\
\hline Ling Yang & Shuanghu Yuan & Lei Zhang \\
\hline China & China & United Kingdom \\
\hline
\end{tabular}


Li Zhang

China

Wei Zhang

China

Yonghui Zhang

China

Shijun Zhang

China

Naiqian Zhao

China

Kaicun Zhao

United Kingdom

Ying-Yong Zhao

USA

Guo-Qing Zheng

China

Guoping Zheng

Australia

Yiwen Zheng

New Zealand

Mingshan Zheng

China

Sihao Zheng

China
Linda Zhong

Hong Kong

Sen Zhong

China

Shanshan Zhou

China

Chunyan Zhou

China

Guang-Zhou Zhou

China

Hong-Hao Zhou

China

Kequan Zhou

USA

Wei Zhou

USA

Lei Zhou

China

Zhiqin Zhou

China

Bing Zhu

China

Liancai Zhu

China
Muhammad Zia-Ul-Haq

Pakistan

Suzanna Zick

USA

Malek Zihlif

Jordan

Lada Zivkovic

Serbia

Denis Zofou

Cameroon

Stefan Zorad

Slovakia

Yuxiao Zou

China

Ping Zou

Japan

Yuan Zou

China

Tycho Zuzak

Germany 